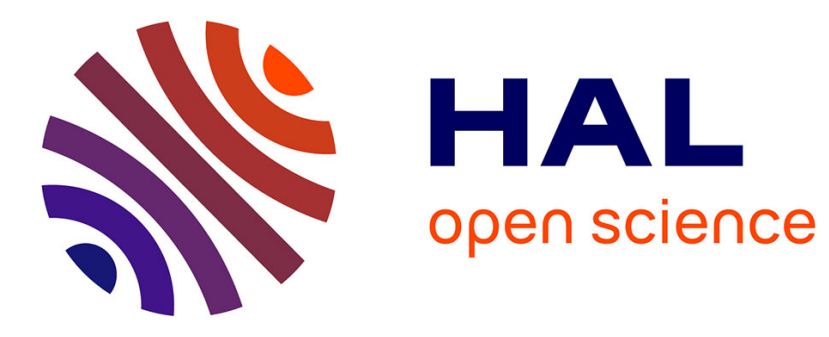

\title{
Fibre multi-wave mixing combs reveal the broken symmetry of Fermi-Pasta-Ulam recurrence
}

Arnaud Mussot, Corentin Naveau, Matteo Conforti, Alexandre Kudlinski, François Copie, Pascal Szriftgiser, Stefano Trillo

\section{To cite this version:}

Arnaud Mussot, Corentin Naveau, Matteo Conforti, Alexandre Kudlinski, François Copie, et al.. Fibre multi-wave mixing combs reveal the broken symmetry of Fermi-Pasta-Ulam recurrence. Nature Photonics, 2018, 12 (5), pp.303-308. 10.1038/s41566-018-0136-1 . hal-02385106

\section{HAL Id: hal-02385106 https://hal.science/hal-02385106}

Submitted on 28 Nov 2019

HAL is a multi-disciplinary open access archive for the deposit and dissemination of scientific research documents, whether they are published or not. The documents may come from teaching and research institutions in France or abroad, or from public or private research centers.
L'archive ouverte pluridisciplinaire HAL, est destinée au dépôt et à la diffusion de documents scientifiques de niveau recherche, publiés ou non, émanant des établissements d'enseignement et de recherche français ou étrangers, des laboratoires publics ou privés. 
archives-ouvertes

\section{Fibre multi-wave mixing combs reveal the broken symmetry of Fermi-Pasta-Ulam recurrence}

Arnaud Mussot, Corentin Naveau, Matteo Conforti, Alexandre Kudlinski, François Copie, Pascal Szriftgiser, Stefano Trillo

\section{To cite this version:}

Arnaud Mussot, Corentin Naveau, Matteo Conforti, Alexandre Kudlinski, François Copie, et al.. Fibre multi-wave mixing combs reveal the broken symmetry of Fermi-Pasta-Ulam recurrence. Nature Photonics, Nature Publishing Group, 2018, 12 (5), pp.303-308. 10.1038/s41566-018-0136-1 . hal02385106

\section{HAL Id: hal-02385106 \\ https://hal.archives-ouvertes.fr/hal-02385106}

Submitted on 28 Nov 2019

HAL is a multi-disciplinary open access archive for the deposit and dissemination of scientific research documents, whether they are published or not. The documents may come from teaching and research institutions in France or abroad, or from public or private research centers.
L'archive ouverte pluridisciplinaire HAL, est destinée au dépôt et à la diffusion de documents scientifiques de niveau recherche, publiés ou non, émanant des établissements d'enseignement et de recherche français ou étrangers, des laboratoires publics ou privés. 


\title{
Fibre multiwave-mixing combs reveal the broken symmetry of Fermi-Pasta-Ulam recurrence
}

\author{
Arnaud Mussot*, Corentin Naveau, Matteo Conforti, Alexandre Kudlinski, Francois Copie, Pascal Szriftgiser* \\ Univ. Lille, CNRS, UMR 8523 - PhLAM - Physique des Lasers Atomes et Molécules, F-59000 Lille, France \\ Stefano Trillo ${ }^{\dagger}$ \\ Department of Engineering, University of Ferrara, Via Saragat 1, 44122 Ferrara, Italy
}

(Dated: December 19, 2017)

\begin{abstract}
Optical fibres constitute a unique ground to investigate one of the most prolific and controversial discovery of modern physics, namely the Fermi-Pasta-Ulam (FPU) recurrence, which becomes manifest as the growth and decay cycles of a comb of sideband pairs from a weakly modulated pump, occurring via modulational instability (MI). To date, however, the genuine spontaneous symmetry-breaking nature of the MI recurrence was never observed (in any area of physics) due to intrinsic limitations of the experimental realizations. In this work, we overcome such limitations by implementing a novel experimental technique which allows us to reconstruct the longitudinal evolution in amplitude and phase of frequency modes via heterodyne detection of the backscattered light. As a result, we clearly observe how the control of the input modulation seed results into qualitatively different types of FPU recursive behavior associated with the spontaneously broken symmetry.
\end{abstract}

The fact that nonlinear systems with several modes exhibit recurrence to the initial state after complex coupling dynamics, instead of equipartition, is known as the FPU paradox $[1,2]$. After several decades of research, the emerging picture is that the equipartition is eventually reached, though over extremely long times, whereas recurrences dominate the behavior over shorter time scales [3]. In dispersive and nonlinear systems that represent the continuous limit of the oscillator chains originally considered by FPU, a similar behavior is envisaged. In particular, in optical fibres where the propagation is ruled by the nonlinear Schrödinger equation (NLSE), FPU recurrence is triggered by the universal phenomenon of MI [4], i.e. the exponential growth of a modulation at the expense of a strong pump. MI induces a seeded modulation frequency to be amplified along with its harmonics via multiwave mixing to form a spectral triangular comb, until the process is reversed leading to FPU recurrence [5]. To date, in single-pass optical experiments, obser-vations are limited to the first recurrence cycle [6,7], whereas the long term dynamics is conjectured to lead to a thermalized state owing to the role played by amplified noise [8].

The investigation of such phenomena are attracting a tremendous interest in optics [9-23], boosted by their strong link with the formation of deterministic breathers [20], statistics of rogue waves [22, 23], supercontinuum generation [21], turbulence [9, 12], and frequency combs in microresonators [10]. Yet, in this context, the essential fact that nonlinear MI does not involve a simple recurrence, but rather a complicated phase-space structure [24-27] associated with spontaneous symmetry breaking [28], was never observed. The signature of such structure is the occurrence of two type of qualitatively different re- currences that are accessible under the same operating conditions. This, however, was eluded so far due to fundamental limitations in the experiments. The major one is represented by the losses which prevents the observation of both type of recurrence, as recently shown in water wave experiments [29] (which are also affected by higher-order effects [30]). An additional limitation, in fibre optics, comes from the need to measure the longitudinal variation of the phases of the mixing products, which turns out to be extremely challenging. In this paper we overcome both of such limitations in a fibre experiment, introducing a (i) loss compensation scheme, and (ii) a novel measurement technique which allows to map the powers and the relevant phases along the fibre. As a result we clearly observe for the first time the signature of the spontaneously broken symmetry of FPU recurrence.

The MI and its recurrent FPU stage are described by the NLSE that rules the propagation of the electric field envelope $E(Z, T)$ in the anomalous dispersion regime of an optical fibre

$$
i \frac{\partial E}{\partial Z}-\frac{\beta_{2}}{2} \frac{\partial^{2} E}{\partial T^{2}}+\gamma|E|^{2} E=0,
$$

where $\gamma$ is the nonlinear coefficient, $\beta_{2}$ is the negative (anomalous) group-velocity dispersion, $Z$ is the distance, and $T$ is the retarded time. According to Eq. (1), MI involves the growth with exponential gain $g(\omega)$ [see Fig. $1(\mathrm{a})$ ] of a modulation with frequency $f_{m}$ (sideband pair with frequency detuning $\pm f_{m}$ ) at the expense of a continuous wave pump with power $P$, provided that the normalized frequency $\omega=2 \pi f_{m} \sqrt{\left|\beta_{2}\right| / \gamma P}$ is such that $\omega \leq 2$. When such instability is seeded, FPU cycles of amplification and back-conversion occur [6, 24, 26, 27]. In order to understand the richness and complexity of 

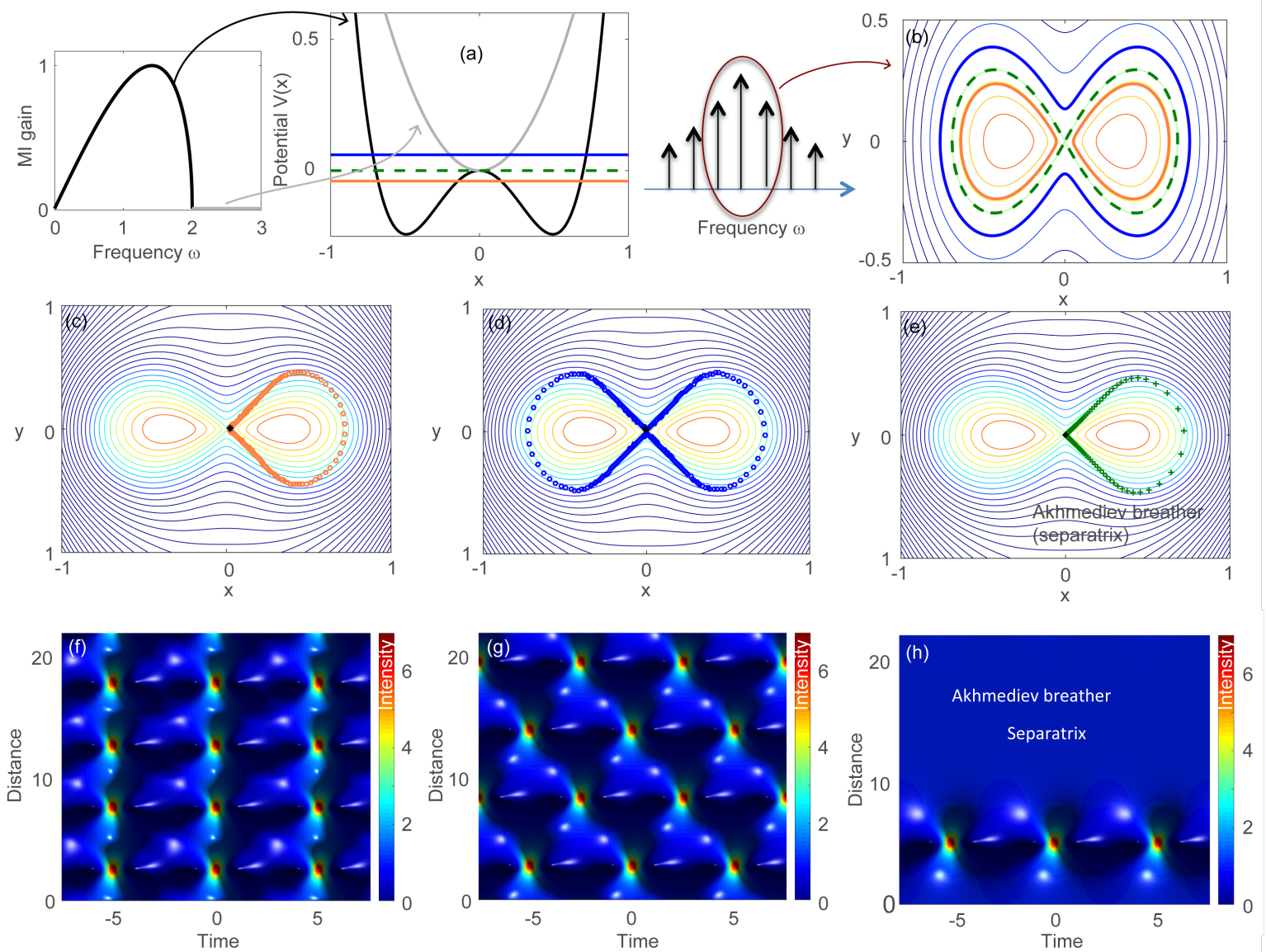

FIG. 1. Spontaneous symmetry-breaking of MI and FPU: (a) MI gain vs. $\omega$ and sketch of the equivalent potential associated to the mixing process, exhibiting spontaneous symmetry breaking (change from single to double well), when going across the onset of MI at $\omega=2$; (b) phase-plane evolutions in the plane $(x, y) \equiv\left(\eta_{1} \cos \Delta \phi, \eta_{1}\right.$ sin $\left.\Delta \phi\right)$, as obtained from the simple oscillator (Hamiltonian in Eq. (2), where $\omega=1.25$ as in the experiment) describing the interaction of the pump and first-order sidebands, as from the schematic drawing of the comb; the separatrix (dashed green line) and the inner and outer orbits (thick orange and blue solid lines) correspond to motions characterized by the total energy levels reported with the same color in (a); (c,d,e) Projections of NLSE trajectories (open dots) on the 3WM phase plane $(x, y)$ for weak initial modulation with different initial phase: (c) $\Delta \phi_{0}=0 ;$ (d) $\Delta \phi_{0}=\pi / 2$; (e) $\Delta \phi_{0}=-0.285 \pi$; (f,g,h) Corresponding full temporal evolutions obtained from the NLSE showing (f) in-phase and (g) out-of-phase recurrences, and (h) the separatrix or Akhmediev breather. In $(\mathrm{f}, \mathrm{g}, \mathrm{h})$ distance and time are in units nonlinear length $Z_{n l}=(\gamma P)^{-1}$ and characteristic time $T_{0}=\sqrt{\left|\beta_{2}\right| Z_{n l}}$, respectively.

the recurrence, the key point is that the onset of MI implies a spontaneous symmetry breaking, as sketched in Fig. 1(a). Indeed the mixing process is equivalent to the motion of an ideal particle in the potential sketched in Fig. 1(a), where the equilibrium in the origin corresponds to the pump wave. Decreasing $\omega$ across the onset of MI, induces the potential to undergo a spontaneous symmetry breaking transition from a single well (with minimum representing the stable pump for $\omega>2$ ) to a double well for $\omega<2$, where the pump exchanges its stability with two new minima. The simplest formalization of this concept, which allows for emphasizing the prominent role of the phases in the dynamics, is the truncation to three-wave mixing (3WM) $[27,31]$, according to which the mixing can be described in terms of a one-dimensional oscillator with Hamiltonian (see methods)

$$
H=\eta_{1}\left(1-\eta_{1}\right) \cos (2 \Delta \phi)+\left(1-\omega^{2} / 2\right) \eta_{1}-3 \eta_{1}^{2} / 4
$$

where $\eta_{1}$ is the power fraction of the first-order $(n=1)$ sidebands and $\Delta \phi=\phi_{0}-\phi_{1}$ is the only effective phase, $\phi_{0}$ and $\phi_{1}$ being the pump and sideband phase, respectively. The level curves of $H$, shown in Fig. 1(b) in the plane $(x, y) \equiv\left(\eta_{1} \cos \Delta \phi, \eta_{1} \sin \Delta \phi\right)$ clearly show the double-loop structure characteristic of the motion in a double-well potential in $x$. The centres or stable points $C_{0}$ and $C_{\pi}$ correspond to the minima of the potential and are the $3 \mathrm{WM}$ representation of invariant modulated waves with equal modulation depth and opposite side-band phase $\Delta \phi=0$ or $\Delta \phi=\pi$ (relative to the pump). In time domain they stand for identical wavetrains except for a temporal shift of half period $\pi / \omega$ due to such shift. 
A separatrix or homoclinic loop (dashed green curve in Fig. 1(b)) divides the possible trajectories into: (i) inner orbits (thick orange curves in Fig. 1(b), corresponding to evolutions in a single well) surrounding only one stable centre, either $C_{0}$ or $C_{\pi}$, thus experiencing phase variations bounded in one (right or left) semiplane; (ii) outer orbits or double-well evolutions (thick blue curve in Fig. $1(\mathrm{~b})$ ), surrounding both stable centres and hence featuring unconstrained phase variations in the whole range $[0$, $2 \pi]$. In particular, in this case, maximum depletion is obtained in alternation between $\Delta \phi=0$ and $\Delta \phi=\pi$, corresponding to temporal wavetrains mutually shifted by half period.

We are interested in the so-called homoclinic crossing phenomenon [24, 26, 27], that is, switching between type (i) and type (ii) orbits controlled by the launching conditions. For a weakly modulated pump, this occurs in the whole range of unstable frequencies, by changing the input phase $\Delta \phi_{0}=\Delta \phi(z=0)$ from amplitude modulation $\Delta \phi_{0}=0$ to frequency modulation $\Delta \phi_{0}=\pi / 2$ (other conditions for switching, though frequency dependent, are discussed in the supplementary information). We show this by means of direct numerical integration of the NLSE. Figure 1(c,d,e) report indeed the projec-tions of the NLSE evolutions on the $3 \mathrm{WM}$ phase-plane for (c) $\Delta \phi_{0}=0 ;$ (d) $\Delta \phi_{0}=\pi / 2$; (e) $\Delta \phi_{0}=-0.285 \pi$ (separatrix). Apart from slight quantitative deviations (due to the neglected higher-order sidebands), the or-bits present exactly the same qualitative features ex-pected from 3WM. The corresponding spatio-temporal evolutions displayed in Fig. 1(f,g,h) show that homo-clinic crossing implies, as expected, switching from the unshifted recurrences shown in Fig. 1(f) to the alter-nance of patterns shown in Fig. $1(\mathrm{~g})$ induced by the shift $\Delta \phi=\pi$, periodically acquired only by the double loop orbits. Finally, the separatrix in Fig. 1(h) repre-sents the well known exact solution of the NLSE known as Akhmediev breather [20, 21, 23, 24, 27].

In order to further show that the qualitatively different FPU recurrences associated with the homoclinic crossing can be detected in an optical fibre, we reported in Fig. 2 further simulations based on the full NLSE with realistic real-world values. We find that, for a very weak input modulation (pump to sideband power ratio of $20 \mathrm{~dB}$, or $\eta_{1}=0.98$ ), nearly $18 \mathrm{~km}$ of optical fibre are necessary to see two recurrences with $\Delta \phi_{0}=0$, as shown in Fig. 2 (a-d), whereas with $\Delta \phi_{0}=\pi / 2$ only one full recurrence is completed over the same length. Indeed, while in both cases two cycles of conversion and back-conversion are achieved in terms of powers [see full spectra in Fig. 2(c,g) or power fractions in Fig. 2(a,e)], when $\Delta \phi_{0}=\pi / 2$ the phase, which spans the whole range $[0,2 \pi]$, recurs only over a double distance. Indeed, in the latter case, the initial condition in phase plane is recovered only after moving through the double loop shown in Fig. 2(h), which require nearly twice the distance needed to move through
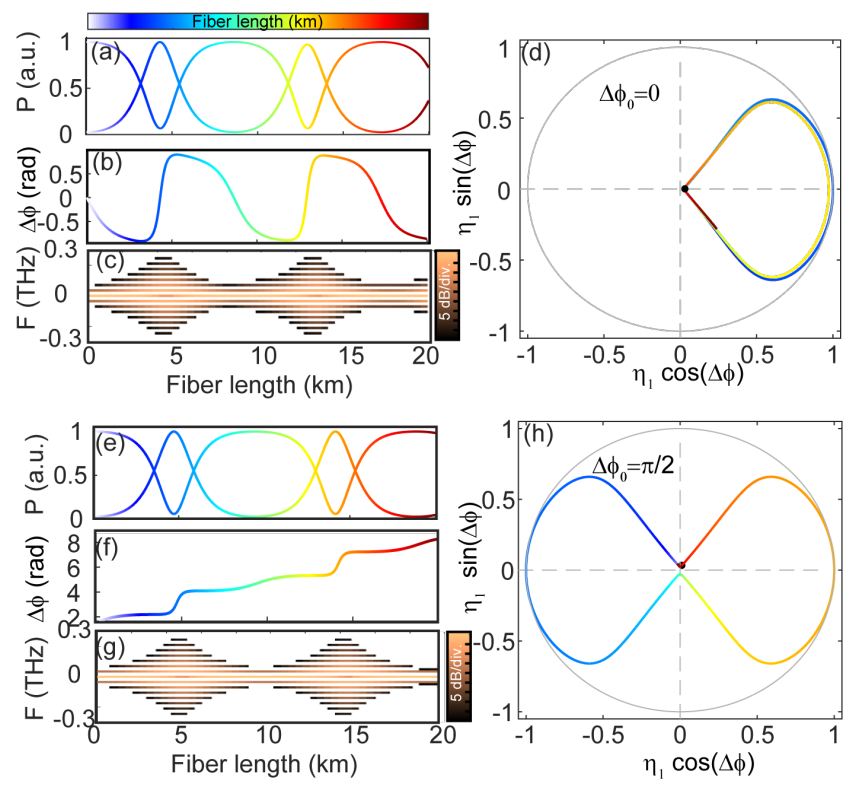

FIG. 2. Homoclinic crossing and period doubling in fibre FPU recurrence: Evolutions along fibre distance of (a,e) pump and sidebands powers; $(\mathrm{b}, \mathrm{f})$ effective phase $\Delta \phi(Z) ;(\mathrm{c}, \mathrm{g})$ full MI power spectrum. $(\mathrm{d}, \mathrm{h})$ phase-plane portrait of the evolution. Upper row (a-d) and lower row (e-h) panels differ only for the input relative phase, set to $\Delta \phi_{0}=0$ (amplitude modulation) and $\Delta \phi_{0}=\pi / 2$ (frequency modulation), respectively. fibre parameters: $\beta_{2}=-19 \mathrm{ps}^{2} / \mathrm{km}$, $\gamma=1.3(\mathrm{~W} \mathrm{~km})^{-1}$, modulation frequency $f_{m}=35 \mathrm{GHz}$, pump power $P=450 \mathrm{~mW}$, pump to signal input power ratio is equal to $20 \mathrm{~dB}$. All power plots are normalized to their respective maxima.

a single loop as in Fig. 2(d). Interestingly, in both cases the weakness of the input modulation induces the conversion process, which is very rapid around the maximum extension of the comb, to strongly slow down near the recurrences (see the plateau in power in Fig. 2(a,e)) when the point passes, in phase-plane, close to the saddle point (i.e., the unstable pump), a feature which is universal for nonlinear oscillators.

In order to have an almost real time access to the ongoing dynamics along the fibre, we have developed a multi-channel vector optical time domain reflectometer (VOTDR). It is optimized for non-linear measurements. Indeed, Rayleigh backscattered light excited from a coherent light source exhibits a jagged appearance due to the fading phenomenon. It originates from the random state of polarization of the scattered light and from a speckle-like phenomenon due to the huge number of scattered waves involved in the process [32]. To overcome this strong limitation, we perform an error correction calibration thanks to a double pulse sequence. A strong signal pulse is first launched inside the experiment fibre (SMF28 ) and a weak reference pulse, typically attenuated by $13 \mathrm{~dB}$, shortly follows. We assume that the weak pulse and its backscattered light experiences similar linear ef- 


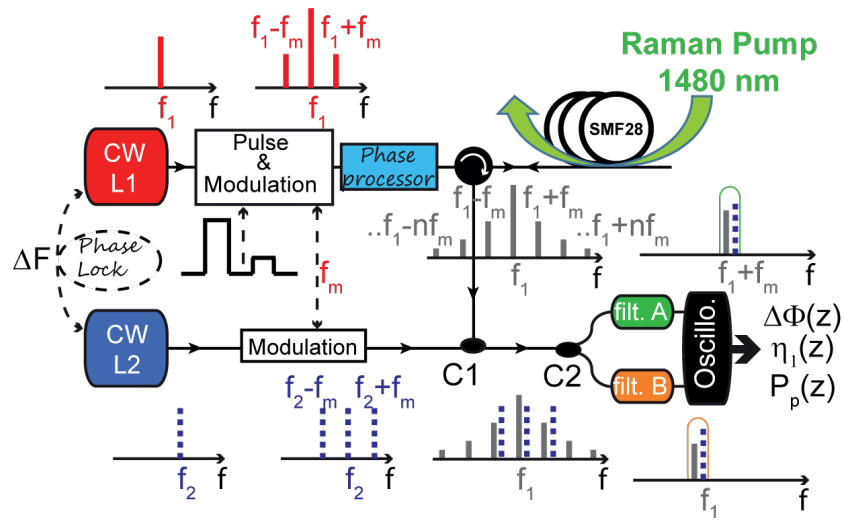

FIG. 3. The novel measurement technique: Basic diagram of the experimental setup. $f_{1,2}$ are the frequencies of the main laser and the local oscillator laser, respectively, with $\Delta F=f_{1}-f_{2}=800 \mathrm{MHz}$. Here $f_{m}=35 \mathrm{GHz}$ is the input modulation frequency (i.e., pump frequency at $f_{1}$, input sideband frequencies at $\left.f_{1} \pm f_{m}\right)$. MI evolution is studied in the $L=7.7 \mathrm{~km}$ long single mode fibre (SMF28). The backscattered signal from the SMF28 goes through a circulator and is analyzed via heterodyning (beating with the local oscillator) and then filtered (filters A and B) to isolate the power and phase evolutions of the pump and the first order sideband in the MI spectral comb.

fects than the strong one, but negligible nonlinear effects. We then correct the amplitude and phase of each strong backscattered wave with those of the weak one. Fig. 3 shows a simplified overview of the experimental setup, see supplementary information for a more thorough discussion and description. After the pulse modulation stage, the incoming light is phase modulated at $f_{m}=35 \mathrm{GHz}$ in order to generate two phase-locked symmetric side bands to initiate the FPU process. Thanks to a commercial Waveshaper, we have a full arbitrary control of the relative phase and intensity of these side bands with respect to the carrier, i.e. the pump. Additionally, along the experiment fibre, a counter-propagating Raman pump (1480 nm) accurately compensates the fibre's linear losses. On the detection side, the Rayleigh backscattered light is mixed with a local oscillator (LO). To perform a coherent heterodyne detection, the $\mathrm{LO}$ is phased locked and detuned by $\Delta F=800 \mathrm{MHz}$ with respect to the pump. In order to provide a local oscillator to each optical component of interest, the LO is also modulated at $f_{m}=35 \mathrm{GHz}$. Since they contain all the information to describe the whole dynamics of our system (see methods), the analysis is restricted to the evolution of the pump and the signal (first order sideband). The time of flight of their beat-note with the modulated LO is isolated with an optical filter, and logged with a real time oscilloscope for further demodulation processing.

In the experiment, the modulation frequency is set to $f_{m}=35 \mathrm{GHz}$, i.e. slightly below the peak MI gain (nonlinear phase matching) frequency at $40 \mathrm{GHz}$, and
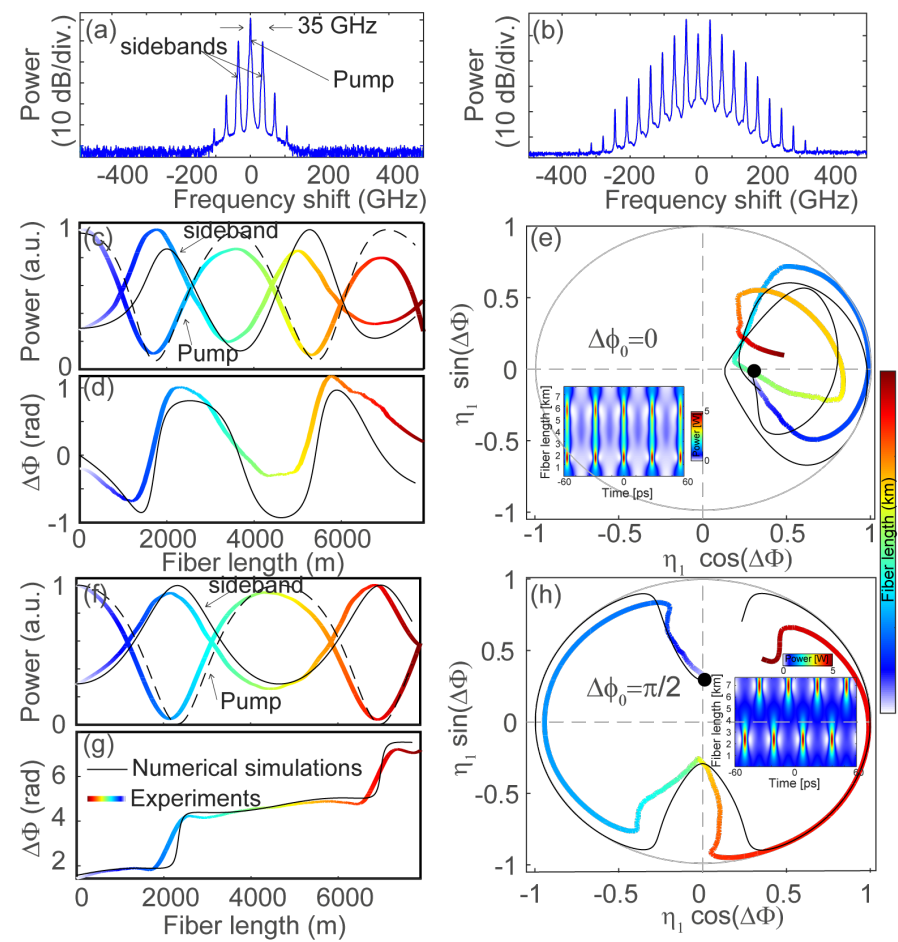

FIG. 4. Observed recurrences and their phase-plane projections: (a,b) spectra at (a) the input of the SMF28 (b) triangular MI spectra at maximal compression point. Evolution along the fibre length of $(c, f)$ the pump power (dashed lines) and the first sideband pair power (dotted lines); (d, g) the relative phase $\Delta \phi(z)$. (e,h) Projections of the evolutions in the $3 \mathrm{WM}$ phase plane (the insets show the corresponding evolutions obtained numerically from the NLSE). Numerical simulations are depicted in black lines and experiments in solid rainbow lines. (c,d,e) and (f,g,h) differ only for the initial relative phase of the modulation $\Delta \phi_{0}=0$ and $\Delta \phi_{0}=\pi / 2$, respectively. Parameters as in Fig. 2 except for $L=7.7 \mathrm{~km}$ and pump-signal power ratio is equal to $8.5 \mathrm{~dB}$. All power plots are normalized to their respective maxima.

such that all the harmonics $n f_{m} n=2,3, \ldots$ are stable, thereby experiencing synchronous growth and decay with the main injected pair [17]. The input spectrum in the SMF28 [see Fig. 4(a)] clearly shows the three main input frequencies, along with very weak $(<-25 \mathrm{~dB})$ harmonics due to residual FWM in the fibers used to carry the signal to the main SMF28 fiber. Figure. 4(b) show the output triangular spectrum under maximal temporal compression. By means of our set-up we have recorded experimentally the longitudinal evolutions of the powers of the pump and of the first-order sideband and their relative phase for two different initial pump-signal relative phase values $\left(\Delta \phi_{0}=0\right.$ and $\left.\Delta \phi_{0}=\pi / 2\right)$. All the evolutions are displayed in Fig. 4(c-h) in solid rainbow lines. Ideally, one should use a very weak modulation at the input. However this results into a large conversion and recurrence distances, which in turn makes the compensation scheme more demanding due to the increased losses 
and enhances the impact of noise amplification due to spontaneous MI, which is ultimately responsible for thermalization [8]. In our experiment, the trade-off between a weak modulation and reasonable fiber length has led us to operate with a pump to sideband power ratio of $8.5 \mathrm{~dB}$ (compared with $20 \mathrm{~dB}$ in the ideal case of Fig. 2), which allowed for scaling down two recurrence periods below the length of $7.7 \mathrm{~km}$ of our fibre. Noteworthy, such a stronger modulation results in a less pronounced plateau in power (see Fig. 4(c,f), and compare with Fig. 4(a,e)), due to passages at larger distances from the saddle point (i.e., the pump) in phase plane. Interestingly enough, by comparing Fig. 4(c) and (d), we notice a nearly perfect recurrence in terms of power levels for $\Delta \phi_{0}=\pi / 2$, whereas the recurrence is within $20 \%$ for $\Delta \phi_{0}=0$, in spite of the same level of Raman pump to compensate for the losses. We mainly attribute this to the fact that, in the presence of strong two sideband modulation, the trajectories followed by the injected amplitude modulation $\left(\Delta \phi_{0}=0\right)$ show a stronger deviation against the ideal case of weak modulation (Fig. 2), compared with the case of the injected frequency modulation $\left(\Delta \phi_{0}=\pi / 2\right)$. This is in-deed evident also from the simulated trajectories (black curves) in Fig. 4(c,f), which highlight the non-ideal be-havior which is also manifest from a slight spatial shift between the dip of the pump and the peak of the side-bands as well as a certain degree of asymmetry between the first cycle and the second, which also reflects in the non perfect superposition of phase-space trajectories in phase-plane (compare Fig. 2(d) and Fig. 4(e)).

Nonetheless we emphasize that the measured data clearly highlight the two qualitatively different type of recurrences. Indeed the range of variation of the phase is bounded between \pm 1 rad for $\Delta \phi_{0}=0$ [see Fig.4(d)], whereas for $\Delta \phi_{0}=\pi / 2$ the phase turns out to span nearly the whole range of $2 \pi$ over a single recurrence [see Fig. 4(g)]. The evidence for the impact of the relative initial phase is even more clear by reconstructing the phase portrait evolutions. For $\Delta \phi_{0}=0$, as shown in Fig. 4(e), the evolution exhibit more than two quasi-periodic orbits spanning only half of the phase plane, as predicted in the ideal scenario (Fig. 2(a-d)). Conversely, for $\Delta \phi_{0}=\pi / 2$, the phase plane evolution in Fig. 4(h) span the whole phase plane making two loops which are nearly symmetric around the vertical axis. All the results are confirmed by numerical simulations of the NLSE (1), with a pretty good agreement (solid black lines). We have also performed additional measurements corresponding to initial conditions located on the other cardinal points of the phase plane, i.e. $\Delta \phi_{0}=\pi, 3 \pi / 2$, which show good agreement with the expected dynamics. Indeed we found that symmetric initial conditions in phase plane, $\Delta \phi_{0}= \pm \pi / 2$ and $\Delta \phi_{0}=0, \pi$, respectively, give almost symmetric orbits in the phase plane (see supplementary information). Finally, we have also provided further evidence for homoclinic crossing by performing additional measurements obtained by varying the phase across the critical value fixed by the slope of the separatrix near the origin (see supplementary information).

We believe that our experimental technique opens new perspectives in the characterization of parametric mix-ing processes in guided wave optics, including the regime of phase-sensitive amplification. On the other hand, our results pave the way towards a more complete understanding of the extremely rich and formidably complex phenomenon of FPU recurrence. In this respect, it must be noticed that most of the recent progresses toward understanding the recurrence and its deterioration have theoretical nature (see e.g. [3, 8, 9] and references therein). Conversely, very few experimental tests exist that deepen the mechanisms of mode interaction and the recurrence in a truly conservative (Hamiltonian) setting, as orig-inally proposed by FPU. Our results establish optical fibres to be a viable platform for investigating all as-pects of mode interactions including the role of the mu-tual phases, at the same time highlighting for the first time the role of spontaneous symmetry breaking and the existence of a complex homoclinic structure in FPU dy-namics ruled by MI. This opens the doors to further experimental investigations that range from the mech-anisms of thermalization and their universality [8, 9], the observation of higher-order separatrices, their associated phase plane dynamics and their role in rogue wave formation [22] and supercontinuum generation, the link with new mechanisms of soliton-mediated recurrences in fibres [33], as well as the transition to chaos induced around the homoclinic structure (homoclinic chaos) in strongly perturbed structures such as fibre passive cavities and microresonators for frequency comb generation [10], where MI remains a key driving mechanism.

\section{METHODS}

Numerics: The simulations reported in Fig. 1-2 are made by integrating the NLSE [Eq. (1)] with pseudospectral or split-step method with typical grid spacing $\Delta T=0.5 \mathrm{ps}$ and $\Delta Z=5 \mathrm{~cm}$. Boundary conditions are implicitly imposed on a temporal windows which take several periods of the modulation period $T_{m}=1 / f_{m}$. The initial condition is set to $E(Z, T)=\sqrt{P_{p}}+$ $\sqrt{P_{s}} \exp \left(-i \Delta \phi_{0}\right)\left[\exp \left(i 2 \pi f_{m} T\right)+\exp \left(-i 2 \pi f_{m} T\right)\right]$, where $P_{p}$ and $P_{s}$ are the pump and sideband power, respectively, which fix the pump to signal power ratio $10 \log _{10} P_{p} / P_{s}$ given in the text $(20 \mathrm{~dB}$ in Fig. 2 or $8.5 \mathrm{~dB}$ in Fig. 4 , corresponding to $P_{s}=4.5 \mathrm{~mW}$ and $P_{s}=63.6 \mathrm{~mW}$, respectively). We have also checked numerically that higher order dispersion terms, as well as Raman effect have a negligible impact on our results and can be neglected, so that the whole dynamics of the system can be accurately captured by the NLSE.

Theory: Starting from the NLSE, the description 
of the nonlinear stage of MI in term of the reduced oscillator (2), can be obtained as follows. The comb that develops from depleted MI is of the form $E(Z, T)=\sqrt{P} \sum_{n} a_{n}(Z) \exp \left(i 2 \pi f_{m} T\right)$, where the sum is over all integers $n=\ldots,-2,-1,0,1,2, \ldots, f_{m}$ is the input modulation frequency and $P$ is the injected (conserved) power, $a_{0}(Z)$ and $a_{ \pm|n|}(Z)$ are the complex amplitudes of the pump and the $n$-th sideband pair, respectively. Since the spectrum is triangular [21] with strong decay between adjacent sidebands of increasing order $\left(\left|a_{n+1}\right|^{2} /\left|a_{n}\right|^{2}(d B)=10 \log _{10}(2-\omega) /(2+\omega)\right.$ at conversion apex, e.g. $\sim-8 \mathrm{~dB}$ at peak gain $\omega=\sqrt{2}[15])$, we can accurately approximate the process by means of three modes ( $3 \mathrm{WM}, n=-1,0,1)$, neglecting higher-order sidebands $|n| \geq 2$ that remain enslaved and phase-locked to the main pair $n= \pm 1$ upon propagation. Hence, further assuming symmetric sidebands $\left(a_{1}=a_{-1}\right)$ as in the experiment, we plug into the NLSE (1) the 3WM ansatz $E(Z, T)=$ $\sqrt{P}\left\{a_{0}(Z)+a_{1}(Z)\left[\exp \left(i 2 \pi f_{m} T\right)+\exp \left(-i 2 \pi f_{m} T\right)\right] / \sqrt{2}\right\}$ and obtain coupled equations by grouping terms at the same frequency. Then, following the approach developed in Refs. [27, 31], we exploit the Hamiltonian structure of the resulting system of equations to reduce them to a one degree of freedom oscillator in terms of the conjugated variables $\eta_{1}=\left|a_{1}\right|^{2}=1-\left|a_{0}\right|^{2}$ and $\Delta \phi=\phi_{0}-\phi_{1}$, which are found to obey the following evolution equations

$$
\begin{aligned}
& \frac{d \eta_{1}}{d z}=\frac{\partial H}{\partial \Delta \phi}=-2 \eta_{1}\left(1-\eta_{1}\right) \sin (2 \Delta \phi), \\
& \frac{d \Delta \phi}{d z}=-\frac{\partial H}{\partial \eta_{1}}=\left(1-\frac{\omega^{2}}{2}\right)-\frac{3}{2} \eta_{1}+\left(1-2 \eta_{1}\right) \cos (2 \Delta \phi),
\end{aligned}
$$

where $z=Z / Z_{n l}$ is the normalized distance in units of the nonlinear length $Z_{n l}=(\gamma P)^{-1}$. Clearly, $H=$ $H\left(\eta_{1}, \Delta \phi\right)$ in Eqs. (3) takes the expression of the Hamiltonian reported in Eq. (2). The invariance of $H$ along the motion allows to draw the level curves reported in Fig. 1(b).

Experiment: Two major challenges faced in the experiment are: (i) the loss compensation in the $7.7 \mathrm{~km}$ long SMF-28, that would induce all the evolutions to drop on phase-shifted evolution [29], thus hiding the broken symmetry of FPU; (ii) to overcome the fading phenomenon that is likely to occur when a quasi-monochromatic wave is launched in an optical fibre [32]. The losses of the SMF-28 are almost perfectly compensated by means of a scheme borrowed by telecommunication systems, which exploits a counter-propaganting wave centred at $1480 \mathrm{~nm}$ acting as a Raman pump (see Fig. 3 and supplemental material for further informations). Concerning the second issue, we remind that a random noise in amplitude and phase is superimposed on the backscattered light originating from variations of the state of the polarisation of the light and/or from local thermo-mechanical fluctu- ations of the scattering volume. We removed the contribution of this detrimental linear phenomenon on the reflected pulse as follows. We launch two consecutive pulses in the fibre. The first one is strong and is responsible for the nonlinear dynamics, whereas a following weaker one $(-13 \mathrm{~dB})$ experiences essentially linear effects. These linear effects are similar to those experienced by the first strong pulse because the time delay between these pulses is extremely short $(1 \mathrm{~ms})$ compared to the characteristic response time of thermo-mechanical fluctuations in the fibre. The amplitude and the phase of the backscattered strong signal is then corrected by means of the weak one. This scheme allows to effectively remove the contribution of the fading effect (the effectiveness of the scheme is further discussed in the supplemental, showing in Fig. S3 a typical trace before and after the correction).

The drawback of this method is that all linear contributions are removed, including the phase due to the group velocity dispersion acquired during the propagation. This contribution is linked to the linear phase mismatch term of the four-photon process underlying the MI process, and can not be neglected. However, it can be easily restored by adding to the compensated phase evolution the characteristic phase term arising from GVD, i.e. $\frac{1}{2} \beta_{2}\left(2 \pi f_{m}\right)^{2} Z$.

Acknowledgments: This work was partly supported by the Agence Nationale de la Recherche through the ANR TOPWAVE, HEAFISY and NoAWE projects, the Labex CEMPI and Equipex FLUX through the "Programme Investissements d'Avenir", by the Ministry of Higher Education and Research, Hauts de France council and European Regional Development Fund (ERDF) through the Contrat de Projets Etat-Region (CPER Photonics for Society, P4S) and FEDER through the HEAFISY project.

Author contributions: A.M. and P.S. have conceived the experimental setup. A.M., C.N., A.K., F.C., P.S. have worked on the experiment. M.C. and S.T. have developed the theoretical aspects. AM, ST, MC and CN performed numerical simulations. All the authors have contributed to analyze the data and write the paper.

* arnaud.mussot@univ-lille1.fr

† ${ }^{\dagger}$ stefano.trillo@unife.it

[1] E. Fermi, J. Pasta, and S. Ulam, in Collected Papers of Enrico Fermi, edited by E. Segré (The University of Chicago, Chicago, 1965), Vol. 2, pp. 977-988.

[2] M.A. Porter, N.J. Zabusky, B. Hu, and D.K. Campbell, Fermi, Pasta, Ulam and the Birth of Experimental Mathematics, American Scientist 97, 214-221 (2009).

[3] M. Onorato, L. Vozella, D. Proment, and Y. V. Lvov, Route to thermalization in the $\alpha$-Fermi-Pasta-Ulam system, PNAS 112, 4208-4213 (2015).

[4] V. E. Zakharov and L. A. Ostrovsky, Modulation instability: The beginning, Physica D 238, 540-548 (2009).

[5] N. Akhmediev, Nonlinear physics - Deja vu in optics, 
Nature 413, 267-268 (2001).

[6] G. Van Simaeys, Ph. Emplit, and M. Haelterman, Experimental demonstration of the Fermi-Pasta-Ulam recurrence in a modulationally unstable optical wave, Phys. Rev. Lett. 87, 033902 (2001); G. Van Simaeys, Ph. Emplit, and M. Haelterman, Experimental study of the reversible behavior of modulational instability in optical fibres, J. Opt. Soc. Am. B 3, 477-486 (2002).

[7] J. Beeckman, X. Hutsebaut, M. Haelterman, and K. Neyts, Induced modulation instability and recurrence in nematic liquid crystals, Opt. Exp. 18, 11185 (2007).

[8] S. Wabnitz and B. Wetzel, Instability and noise-induced thermalization of Fermi-Pasta-Ulam recurrence in the nonlinear Schrödinger equation, Phys. Lett. A 378, 27502756 (2014).

[9] M. Guasoni, J. Garnier, B. Rumpf, D. Sugny, J. Fatome, F. Amrani, G. Millot, and A. Picozzi, Incoherent FermiPasta-Ulam Recurrences and Unconstrained Thermalization Mediated by Strong Phase Correlations, Phys. Rev. X 7, 011025 (2017).

[10] C. Bao, J. A. Jaramillo-Villegas, Y. Xuan, D. E. Leaird, M. Qi, and A. M. Weiner, Observation of Fermi-PastaUlam Recurrence Induced by Breather Solitons in an Optical Microresonator, Phys. Rev. Lett. 117, 163901 (2016).

[11] M. Närhi, B. Wetzel, C. Billet, S. Toenger, T. Sylvestre, J.-M. Merolla, R. Morandotti, F. Dias, G. Genty, and J. M. Dudley, Real-time measurements of spontaneous breathers and rogue wave events in optical fibre modulation instability, Nature Commun. 7, 13675 (2016).

[12] J. M. Soto-Crespo, N. Devine, and N. Akhmediev, Integrable Turbulence and Rogue Waves: Breathers or Solitons?, Phys. Rev. Lett. 116, 103901 (2016).

[13] B. Kibler, A. Chabchoub, A. Gelash, N. Akhmediev, and V. E. Zakharov, Super-regular Breathers in Optics and Hydrodynamics: Omnipresent Modulation Instability beyond Simple Periodicity, Phys. Rev. X 5, 041026 (2015).

[14] S. Toenger, T. Godin, C. Billet, F. Dias, M. Erkintalo, G. Genty, and J. M. Dudley, Emergent rogue wave structures and statistics in spontaneous modulation instability, Sci. Rep. 5, 10380 (2015).

[15] A. Bendhamane, A. Mussot, P. Szriftgiser, A. Kudlinski, M. Conforti, S. Wabnitz, S. Trillo, Optimal frequency conversion in the nonlinear stage of modulation instability, Opt. Exp. 23, 30861-30871 (2015).

[16] G. Biondini and D. Mantzavinos, Universal Nature of the Nonlinear Stage of Modulational Instability, Phys. Rev. Lett. 116, 043902 (2016).

[17] S. A. Chin, O. A. Ashour, and M. R. Belić, Anatomy of the Akhmediev breather: Cascading instability, first formation time, and Fermi-Pasta-Ulam recurrence, Phys. Rev. E 92, 063202 (2015).

[18] A. Mussot, A. Kudlinski, M. Droques, P. Szriftgiser, and N. Akhmediev, Fermi-Pasta-Ulam recurrence in nonlinear fibre optics: the role of reversible and irreversible losses, Phys. Rev. X 4, 011054 (2014).

[19] M. Erkintalo, K. Hammani, B. Kibler, C. Finot, N. Akhmediev, J. M. Dudley, and G. Genty, Higher-Order Modulation Instability in Nonlinear fibre Optics, Phys. Rev. Lett. 107, 253901 (2011).

[20] B. Kibler, J. Fatome, C. Finot, G. Millot, F. Dias, G. Genty, N. Akhmediev, and J. M. Dudley, The Peregrine soliton in nonlinear fibre optics, Nature Phys. 6, 790-795 (2010).

[21] J. M. Dudley, G. Genty, F. Dias, B. Kibler, and N. Akhmediev, Modulation instability, Akhmediev Breathers and continuous wave supercontinuum generation, Opt. Exp. 17, 21497-21508 (2009).

[22] M. Onorato, S. Residori, U. Bortolozzo, A. Montina, and F. T. Arecchi, Rogue waves and their generating mechanisms in different physical contexts, Phys. Rep. 528, 47-89 (2013).

[23] J. M. Dudley, F. Dias, M. Erkintalo, and G. Genty, Instabilities, breathers and rogue waves in optics, Nature Photon. 8, 755-764 (2014).

[24] N. N. Akhmediev, V.M. Eleonoskii, and N.E. Kulagin, Generation of periodic trains of picosecond pulses in an optical fibre: exact solutions, Sov. Phys. JETP 62, 894 (1985); N.N. Akhmediev, V.M. Eleonskii, and N. E. Kulagin, Exact first-order solutions of the nonlinear Schrödinger equation, Theor. Math. Phys. (USSR) 72, 809 (1987).

[25] M. J. Ablowitz and B. M. Herbst, On Homoclinic Structure and Numerically Induced Chaos for the Nonlinear Schrödinger Equation, SIAM J. Appl. Math. 50, 339 (1990);

[26] H. T. Moon, Homoclinic Crossings and Pattern Selection, Phys. Rev. Lett. 64, 412 (1990).

[27] S. Trillo and S. Wabnitz, Dynamics of the modulational instability in optical fibres, Opt. Lett. 16, 986 (1991).

[28] C. Liu, Spontaneous symmetry breaking and chance in a classical world, Phil. Science 70, 590-608 (2003).

[29] O. Kimmoun, H.C. Hsu, H. Branger, M.S. Li, Y.Y. Chen, C. Kharif, M. Onorato, E. J. R. Kelleher, B. Kibler, N. Akhmediev, and A. Chabchoub, Modulation Instability and Phase-Shifted Fermi-Pasta-Ulam Recurrence, Sci. Rep. 6, 28516 (2016).

[30] M. J. Ablowitz, J. Hammack, D. Henderson, and C.M. Schober, Modulated periodic Stokes waves in deep water, Phys. Rev. Lett. 84, 887-890 (2000).

[31] G. Cappellini and S. Trillo, Third-order three-wave mixing in single-mode fibres: exact solutions and spatial instability effects, J. Opt. Soc. Am. B 8, 824 (1991).

[32] P. Healey, Fading in heterodyne OTDR, Electron. Lett. 20, 30-32 (1984).

[33] G. Deng, S. Li, G. Biondini, and S. Trillo, Recurrence due to periodic multisoliton fission in the defocusing nonlinear Schrödinger equation, Phys. Rev. E 96, 052213 (2017).

[34] See Supplemental Material for further details on the experimental setup, the connection to exact solutions of the NLSE, and additional measurements. 